\title{
Anti-Aging Efficacy of a New Alendronate-Pravastatin Cosmetic Combination: A Randomized Double Blind Comparative Study
}

\author{
Bénédicte Cantecor ${ }^{1,2,3}$, Marie-Pierre Savelli ${ }^{2}$, Philippe Piccerelle ${ }^{2,4}$, Nicolas Lévy ${ }^{1,5}$, \\ Vincent Bonniol ${ }^{1,6}$, Pierre Cau ${ }^{1,3}$ \\ ${ }^{1}$ Inserm UMR_S 910, Aix-Marseille University, Marseille, France; ${ }^{2}$ Pharmaco-Technology Laboratory, Aix-Marseille University, \\ Marseille, France; ${ }^{3}$ Cell Biology Laboratory, La Timone Hospital, Marseille, France; ${ }^{4}$ Mediterranean Institute of Marine and Terres- \\ trial Biodiversity and Ecology (IMBE), Aix-Marseille University, Marseille, France; ${ }^{5}$ Human Genetics Department, La Timone Hos- \\ pital, Marseille, France; ${ }^{6}$ Prenyl B Laboratory, Marseille, France. \\ Email: pierre.cau@univ-amu.fr
}

Received June $7^{\text {th }}$, 2013; revised July $9^{\text {th }}$, 2013; accepted July $17^{\text {th }}, 2013$

Copyright (c) 2013 Bénédicte Cantecor et al. This is an open access article distributed under the Creative Commons Attribution License, which permits unrestricted use, distribution, and reproduction in any medium, provided the original work is properly cited.

\begin{abstract}
Progeria is a rare genetic disease that causes accelerated aging and death in children at a mean age of 13.5 years. An aminobisphosphonate-statin combination has been shown to reduce the toxicity of the mutated protein, progerin, in progeria patient cell cultures and in a mouse model of the disease. This combination is currently being tested in a European Therapeutic Trial for progeria in Marseille (ClinicalTrials.gov identifier NCT00731016). Progerin has been shown to be produced by skin cells during physiological aging. The objective of this study was to assess the efficiency of a new and original cosmetic formulation containing alendronate and pravastatin sodium salts, reduce crow's feet wrinkles, and cheek hollow in a double blind, randomized and placebo controlled comparative study. Three cosmetic preparations were evaluated using Fast Optical in vivo Topometry of human Skin (FOITS): one containing sodium alendronate and sodium pravastatin, a placebo, and a commercial anti-aging product. Fifty-seven female and twenty-five male volunteers between 51 and 71-year-old were selected. Each subject tested two of the three products once a day, in the evening, by spreading each selected product on one side of the face. Skin micro-relief was analyzed at 0, 28, 56 and 84 days. Statistical analysis of 7 clinical qualitative (left or right side of face, gender, and 3 skin types) and 6 quantitative parameters (age, weight at each test time, wrinkle clinical grade at inclusion time) showed no statistical differences between the three tested products. In contrast, most of the 8 quantitative FOITS parameters describing skin micro-relief were statistically improved by the alendronate-pravastatin combination compared to the placebo or to the commercial anti-aging product. A cosmetic preparation containing alendronate and pravastatin sodium salts exhibited anti-aging effects by reducing crow's feet wrinkles and restoring cheek volume.
\end{abstract}

Keywords: Skin Aging; Progerin; Statin; Aminobisphosphonate; Double Blind Comparative Clinical Study; Placebo; FOITS

\section{Introduction}

The human life span has more than doubled over the last two centuries. With this increase in life expectancy, a growing segment of the aging population seeks to preserve a youthful appearance for as long as possible [1]. For thousands of years cosmetic products have been used around the world to improve the physical appearance and hide flaws. In response to growing consumer demand in this sector, numerous companies have developed active "anti-aging" ingredients, which are claimed to have to- pical anti-aging effects. These active ingredients are often well known molecules (e.g., vitamin A or C, glycolic acid, etc.), which have been used for many years to reduce the signs of aging [2].

Research on progeria provides a new source of active anti-aging ingredients. Progeria, also known as Hutchinson-Gilford Progeria Syndrome (HGPS), is one of 7000 identified rare diseases [3]. Progeria is characterized by premature and accelerated aging in children. The first signs of the disease appear in the first $12-18$ months of 
life and are characterized by a break in the growth curve. The average age of death is 13.5 years, often caused by myocardial infarction or stroke. Progeria children have a characteristic phenotype, including very thin skin revealing superficial veins, pinched nose, alopecia and microretrognathia. Progeria children exhibit severe generalized atherosclerosis and suffer from a significant reduction in bone density and lipoatrophy $[3,4]$.

The mutation responsible for progeria occurs in the LMNA gene, which encodes lamins A or C through alternative splicing, and was identified independently in 2003 by us [5] and a US research team [6]. Lamins $A$ and $C$ are nuclear intermediate filaments. Lamin A is synthesized as a precursor, prelamin $\mathrm{A}$, whose posttranslational processing involves four successive steps. A cytosolic farnesyl-transferase binds a 15-carbon isoprenoid group called farnesyl to the cysteine (C) in the C-terminal CaaX box of prelamin $\mathrm{A}$, where aa are two aliphatic residues and $\mathrm{X}$ is methionine. The farnesyl group allows prelamin A to be anchored into the cytosolic leaflet of the endoplasmic reticulum (ER) envelope, where prelamin A can be processed by ER enzymes, whoses active sites face the cytosol. Next, a protease (FACE2/Rce1 or FACE1/ ZMPSTE24) cleaves the last 3 residues, aaX. The farnesylated cysteine is then carboxymethylated. Finally, FACE1/ZMPSTE24 cleaves the last prelamin A 15 C-terminal residues, including the farnesylated cysteine, giving the "mature" soluble lamin A. This protein is further imported through nuclear pore complexes into the nucleoplasm, where it exhibits two different localizations: in the nuclear lamina, close to the nucleoplasmic face of nuclear envelope; and as a component of the nuclear matrix in the nucleoplasm [7].

The progeria mutation activates a cryptic splicing site, which leads to the synthesis of an mRNA deleted of 150 nucleotides that is translated into a protein deleted of 50 residues called progerin. Because the deletion encompasses the FACE1/ZMPSTE24 recognition site, this protease cannot clip off the last $15 \mathrm{C}$-terminal amino acids of the laminA precursor. Progerin retains the farnesyl group that anchors it to membranes and enables it to accumulate in the nuclear lamina after nuclear import. The same pathophysiological mechanism, i.e., the persistance of farnesylatedprogerin or prelamin A, has been observed in three different genetic diseases, including HGPS, restrictive dermopathy (RD) and mandibuloacral dysplasia, resulting in accelerated aging [7].

The accumulation of farnesylatedprogerin/prelamin A in the nuclear lamina induces both the characteristic changes in nuclear shape and size and a decrease in the amount of "soluble" mature lamin A in the remaining nucleoplasm. This abnormality in the composition of the nuclear matrix causes disorders in several nuclear ge- nome activities (e.g., DNA repair, RNA transcription and maturation, etc.) that trigger cell, and therefore organismal, aging.

Progerin is also produced by cells from aged subjects without LMNA mutations by age-related dysfunction of the mRNA splicing machinery via the cryptic splicing site in exon 11 of the pre-mRNA. Progerin is probably a pro-aging factor [8]. The expression of progerin or farnesylatedprelamin A by stem cells from adult tissues belonging to either epidermal [9] or mesenchymal [10] lineages leads to rapid exhaustion of the stem cell pool, another pro-aging event. Finally, progerin may be a marker of skin aging because progerin was shown to be expressed by both epidermal keratinocytes and dermal fibroblasts in skin biopsies from aged subjects [11].

We showed that a combination of two drugs, an aminobisphosphonate and a statin, decreases the accumulation and/or persistence of the prenylated nuclear proteins responsible for cell aging in progeria patients. The same combination also corrects several biological and skin disorders (e.g., alopecia, subcutaneous lipoatrophy) in a mouse model of human progeria and increases the life span of these mice [12].

Previously, a synergistic combination of an aminobisphosphonate (sodium alendronate, Aln) and a statin (sodium pravastatin, Pra) was incorporated into a cosmetic oil-in-water emulsion product with the required characteristics for topical application. A preliminary in vitro permeability study using this cosmetic vehicle revealed that both molecules were able to diffuse through the skin barrier into the dermis in small amounts despite their high hydrophilicity and strong ionization [13].

In this study, we present the results of a placebo-controlled double blind study using fringe projection and show that the cosmetic preparation containing alendronate and pravastatin sodium salts reduces crow's feet and restores cheek volume, both of which are characteristics of physiological skin aging.

\section{Subjects and Methods}

\subsection{Subject Selection}

\subsubsection{Inclusion Criteria}

Eighty-two healthy participants between 51 and 71year-old (57 females, 25 males) were enrolled in the study. They exhibited a caucasian healthy skin type on the anatomic area studied.

The subjects displayed wrinkles and/or fine lines on the crow's feet with a grade from 3 to 5 for the women and from 4 to 6 for the men [14], as well as hollow cheeks. Weight remained stable for at least 3 months (variation, less than $2.0 \mathrm{~kg}$ ).

A homogeneous distribution was ensured between 
each group (each group represented the testing of one product on one side of the face) for the following criteria: skin type (dry or combination skin), gender (female or male), age, and wrinkle clinical grade.

\subsubsection{Exclusion Criteria}

All participants were dedicated to the present study and signed an informed consent form. Subjects were excluded when they were unable to comply with the following protocol requirements: body weight variation less than 2 kg during the study time course; for female subjects, neither enter into, nor change or stop, a hormone replacement therapy; no entry into a dehydroepiandrosterone (DHEA)-based treatment; no changes in sport habits; no modification of way of life; no use of products, techniques or surgery with an anti-wrinkle action; no sunbathing.

\subsection{The Three Topical Products}

Of the three products tested, two were oil-in-water $(\mathrm{O} / \mathrm{W})$ emulsions: one emulsion contained sodium alendronate and sodium pravastatin (Aln-Pra); the second emulsion was the corresponding drug-free placebo. Both possessed a light, non-oily texture that is easy to apply, penetrates well and is suited for all skin types. Therefore, the emulsions exhibited the characteristics that influence consumer acceptance and subject compliance (i.e., visual appearance, odor and residual impression after application) for a cosmetic product [15].

The $\mathrm{O} / \mathrm{W}$ emulsions were prepared by stepwise addition of the inner oily phase to the aqueous phase at $85^{\circ} \mathrm{C}$. The addition of oil was performed under rotor stator agitation at $1700 \mathrm{rpm}$ (Turbotest, VMI Rayneri, France). The chemical products used in the formulations are listed elsewhere [13]. The final concentrations of alendronate and of pravastatin in the cosmetic product were adjusted from the transdermal delivery measurements [13], to obtain a margin of safety of $>100$, in accordance with European guidelines [13]. The final concentration of Aln and Pra was not detailed for industrial privacy.

The Aln-Pra containing cosmetic and its placebo were compared to a third product (Cosmetics X), a commercial anti-aging cosmetic product. Cosmetics $\mathrm{X}$ contains the following ingredients: water, hydrogenated polyisobutene, glycerin, cyclohexasiloxane, shea butter, poly C10-30 alkyl acrylate, aluminum starch octenylsuccinate, sucrose stearate, beeswax, stearic acid, triethanolamine, dimethicone, dimethylconol, caffeine, sodium cocoyl glutamate, sodium hydroxide, sodium polyacrylate, silica, palmitic acid, vignaaconitifolia seed extract, hydrolyzed soy protein, caprylic/capric triglyceride, acetyl trifluoromethylphenylvalylglycine, crithmummaritimum extract, oxothiazolidine-carboxylic acid, acrylates/C10-30 alkyl acry- late crosspolymer, retinylpalmitate, pentaerythrityl tetradi-t-butyl hydroxyhydrocinnamate, methylparaben, phenoxyethanol, chlorphenesin, ethylparaben, linalool, geraniol, alpha-isomethyl ionone, amyl cinnamal, limonene, citronellol, butylphenylmethylpropional, hexyl cinnamal, benzyl alcohol, benzyl benzoate, benzyl salicylate, fragrance.

\subsection{Product Application in a Double Blind Protocol}

Neither the participating subjects nor the investigator were aware of the product type being applied throughout the study. The subjects served as their own controls for product comparison in time. The study lasted for 84 days following the first application of the products.

The study took place between mid-November and mid-February in the north of France. Application of any anti-wrinkle cosmetic product was prohibited two weeks prior to the start of the study. During the three months of the study, the subjects did not apply other cosmetic products to the studied areas (only the usual cleanser, the usual moisturizing cream in the morning, and make up for the lips, eyes and blusher powders were accepted).

Each subject applied one product on each side of the face (randomly chosen at the start of the study) after washing and drying, once daily in the evening. The applied quantities corresponded to normal conditions of use. On the day of the measurements, no other cosmetic products were used (only facial cleanser with water was accepted). The two products tested by each subject were randomly selected.

\subsection{Skin Micro-Relief Measurements}

Evaluation was carried out on day 0 and after 28, 56 and 84 days of treatment. In a dark room, the subjects wore a mobcap on their head, kept the eyes open and looked straight ahead. The positioning of the sensor and of the subject were made easier with the use of a measurement bench (VisioFace ${ }^{\circledR}$ ), which enabled the face to be kept in the same position and provided reproducible positioning of the sensor throughout the study.

\subsubsection{Fringe Projection on Crow's Feet: Rugosity, Area and Volume Parameters}

The Fast Optical in vivo Topometry of human Skin (FOITS) technique allows modifications in the skin microtopography to be quantified $[16,17]$. The measurements were taken using an optical system dedicated to the metrology of the skin surface relief. The measuring sensor comprised a projector and a high-resolution CCD camera (Dermatop system, Breukmann, Germany; field of view: $30 \times 40 \mathrm{~mm}$; averaged lateral resolution: $32 \mu \mathrm{m}$ ) 
and Optocat acquisition software (EoTech, France). The wrinkles in both crow's feet were measured separately. The visualization of the initial measurement on the screen (at day 0) ensured a good repositioning of the studied area on days 28, 56 and 84 .

Skin surface topography was analyzed by calculating the standard roughness parameters, which were extracted from a surface of $43 \times 32 \mathrm{~mm}\left(12 \mathrm{~cm}^{2}\right)$. Fringe projection data analysis was carried out using both the TopoSurf and Optocat analysis systems. The principle involves quantifying the micro-relief of the studied area by analyzing the deformation of a network of high contrast fringe lines.

The rugosity parameters were quantified on a sample of profiles perpendicular to the wrinkles and fine lines on the area of interest:

- SQ: roughness with regard to the average quadratic variation $(\mathrm{mm})$. Averaged variations in amplitude of the relief integrated into the studied surface. An SQ decrease indicates smoothing of the surface and a decrease in the wrinkles and fines lines.

- ST: maximum amplitude of the relief (mm). An ST decrease shows a reduction in the main wrinkle.

- SA: averaged roughness (mm). Averaged variations in amplitude of the relief integrated into the studied surface. An SA decrease evidences smoothing of the surface and a decrease in wrinkles and fine lines.

- Stm: mean difference between peaks and valleys (mm). An Stm decrease indicates smoothing of the studied surface.

The area and volume (morphology) parameters were also quantified-wrinkles and fine wrinkles were detected after the use of several filters, and a polynomial correction for flattening of the area of interest was applied:

- Ar: Area of the main wrinkle $\left(\mathrm{mm}^{2}\right)$.

- Vol: Volume of the main wrinkle $\left(\mathrm{mm}^{3}\right)$.

\subsubsection{Fringe Projection: Cheek Volume}

Fringe projection technology, adapted to the analysis of face morphology, allows the characterization of the cheek morphology before (day 0) and after treatment (days 56 and 84). The 3 dimensional (3D) measurements were carried out using the fringe projection system (halogen projector) coupled to a high-resolution CCD camera (black and white $-768 \times 512$ pixels; field of view: $360 \times$ $\left.210 \times 270 \mathrm{~mm}^{3}\right)$ (EoTech, France) linked to Optocat acquisition software (EoTech, France). The lighting system coupled to a camera supply enabled the projection of a sequence of lattice lines onto the object (fringes). Threedimensional information regarding the object was calculated from the deformation of the fringes on the object's surface and recorded by the digital camera. Both cheeks were the analyzed separately.
Analysis of these 3D acquisitions consisted of matching the envelope of the studied area obtained at different times with the kinetics of the envelope determined at the initial time. Then a region of interest (ROI) was defined on the studied area (cheek). This ROI, located at the same position for all acquisitions from a given subject, allowed the volume within the ROI (relative Volume, relVol, in $\mathrm{mm}^{3}$ ) and its projection in the reference plane (Standardized relative Volume: StrelVol) to be calculated (Optocat software, EoTech, France). An increase in the relVol indicated cheek "filling" in response to the cosmetics.

\subsubsection{Data Analysis and Statistics}

XLSTAT $^{\circledR}$ software (AddinSoft) was used for statistical analysis and box plot drawings. Rugosity and morphology parameters were compared between groups using the Kruskall-Wallis test. Comparison between groups with discriminant analysis was performed using Roy's greatest root test [18]. Percentage changes for each parameter ([D56-D0]/D0, [D84-D0]/D0 and [D84-D56]/D56) were calculated using mean values. $\mathrm{P}$ values $<0.05$ were considered statistically significant.

\section{Results and Discussion}

Multiparametric discriminant analysis showed no statistical difference $(p=0.458)$ between the three treatment groups (Figure 1) for the six quantitative and six qualitative clinical parameters. In contrast, discriminant analysis using changes in rugosity and morphological parameters during the study time-course revealed a statistical difference $(p=0.022)$ between the three treatment groups (Figure 2).

The changes in both morphological and rugosity parameters during the study time-course are shown in Figure 3.

The topical application of the placebo preparation, which lacked the active drugs, showed no improvement in rugosity parameters with time ( $\pm 5 \%$ changes) or morphological parameters ( $+20 \%$ in both volume and area of main wrinkle; $\pm 5 \%$ in relative volume, relVol, and standardized relative volume, StrelVol, of the hollow cheek).

The commercial cosmetic also showed no improvement in rugosity and morphological skin parameters.

Interestingly, topical application of a product containing the alendronate-pravastatin combination for 56 days improved the rugosity parameters, ST, SA and SQ (in the range $-5 \%$ to $-10 \%$ ) and the morphological parameters, Vol and Area (-20\%), which were stable after 84 days of treatment (Figure 4). Cheek morphological parameters (relative Volume, relVol, and Standardized relative Volume, StrelVol) were improved $(-8 \%$ and $-10 \%$, respectively) after 84 days of topical alendronate-pravastatin 


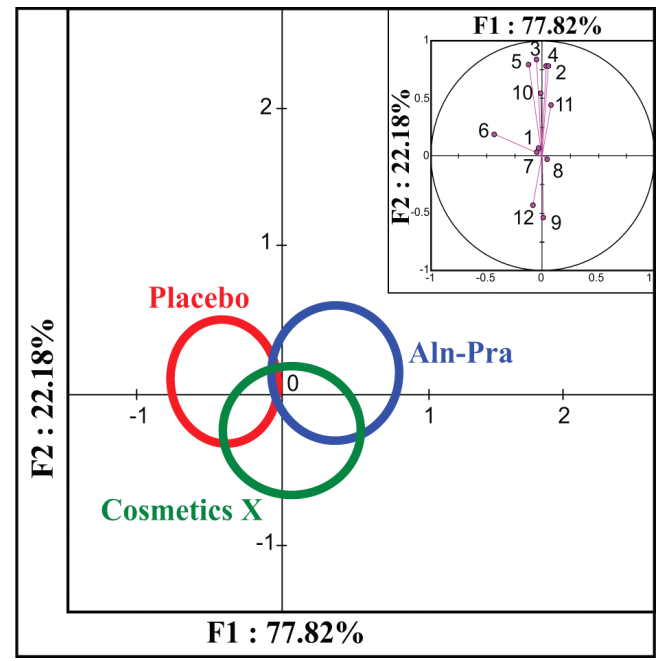

Figure 1. Discriminant analysis shows no statistical differences between the three treatment groups according to six quantitative and six qualitative clinical parameters. The 95\% confidence circle around the means delineates the three treatment groups. The perpendicular axes describe the combined variance of the parameters analyzed (F1 and F2 axis $=100 \%)$. The contributions of each parameter to the variances on the $X$ and $Y$ axes are shown in the insets. Inset: The six quantitative parameters: 1 , age at day $0 ; 2$, weight at day $0 ; 3$, weight at day $28 ; 4$, weight at day $56 ; 5$, weight at day $84 ; 6$, wrinkle grade at day 0 . The six qualitative parameters: 7 , left side analysis; 8 , right side analysis; 9 , female gender; 10, male gender; 11, combination skin type; 12, dry skin type.

treatments.

The improvement in aging-related skin micro-relief produced by the alendronate-pravastatin combination results from several mechanisms. The percutaneous absorption of alendronate has already been shown to be increased by fatty acids [19], some of which are components of our cosmetic excipient [13]. The percutaneous absorption of the alendronate-pravastatin combination has already been demonstrated [13].

Alendronate has been shown to act both extracellularly and intracellularly. Indeed, alendronate binds to the extracellular domain of connexin 43 expressed by keratinocytes [20] and dermal fibroblasts [21, 22].

In osteoblasts and osteocytes, activation of the SrcERK signaling pathway by alendronate results in cell survival [23]. Alendronate may elicit the same effect in skin cells. Furthermore, alendronate has been shown to enter cells through fluid-phase endocytosis [24], to inhibit lysosomal $\mathrm{H}^{+}$-ATPase and several protein tyrosine phosphatases [25], and to activate the PI3K/NFkappaB signaling pathway. However, the main activity of alendronate and of other aminobisphosphonates is to inhibit the farnesylpyrophosphate synthase [26,27], thus impairing the isoprenoid biosynthesis pathway whose two products,

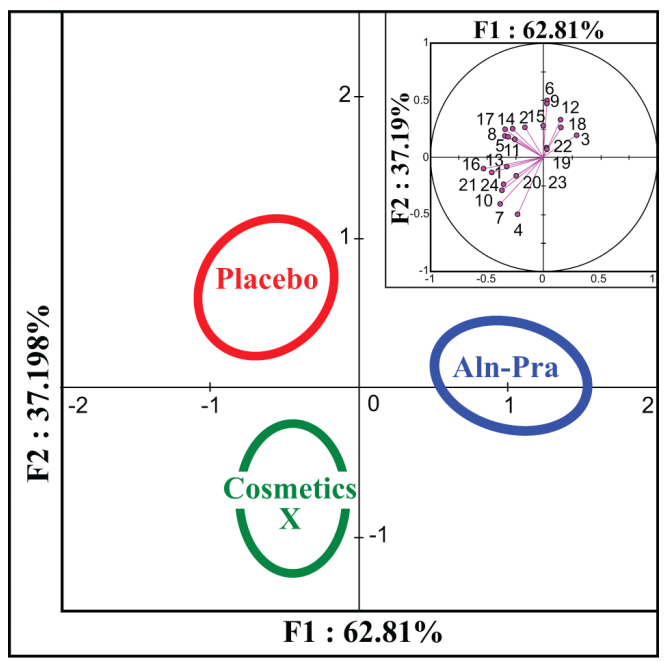

Figure 2. Discriminant analysis shows statistical differences for percentage changes in the four rugosity parameters and the four morphological parameters between the three treatment groups. The $95 \%$ confidence circle around the means delineates the three treatment groups. The perpendicular axes describe the combined variance of the parameters analyzed (F1 and F2 axis $=100 \%)$. The contribution of each of the 24 parameters to the variances on the $X$ and $Y$ axes are shown in the insets. Percentage changes in rugosity parameters: 1, 4, 7, 10: [D56-D0]/D0. 2, 5, 8, 11: [D84-D0]/D0. 3, 6, 9, 12: [D84-D56]/D56. 1, 2, 3: ST (maximum amplitude of the relief). 4, 5, 6: SA (average roughness). 7, 8, 9: SQ (average roughness with regard to the average quadratic variation). 10, 11, 12: Stm (mean difference between peaks and valleys). Percentage changes in morphological parameters: 13, 16, 19, 22: [D56-D0]/D0. 14, 17, 20, 23: [D84-D0]/ D0. 15, 18, 21, 24: [D84-D56]/D56. 13, 14, 15: Vol (volume of the main wrinkle). 16, 17, 18: Ar (area of the main wrinkle). 19, 20, 21: relVol (relative volume of the hollow cheek). 22, 23, 24: StrelVol (standardized relative volume of the hollow cheek).

farnesyl and geranylgeranyl groups, anchor several proteins to membranes, including the biomarker of skin aging, progerin [11]. In bone and cartilage tissues, alendronate has been shown to inhibit the synthesis and activity of various matrix metalloproteases (MMP), thus decelerating collagen and proteoglycan degradation.

Skin keratinocytes and fibroblasts also express several MMPs [28,29]. Skin fibroblasts and keratinocytes synthesized several proteoglycans [30] that contribute to, e.g., skin volume and hydration [31,32].

The pharmacokinetics of statins are best understood in hepatocytes [33,34]. Regarding skin keratinocytes and fibroblasts, several plasma membrane transporters of the OATP family [35], allow statin entry [36,37], whereas ATP-driven transporters of the MRP family [38,39] are involved in statin efflux [40,41]. Besides their pleiotropic effects observed in various dermatological conditions [42], statins are also inhibitors of the isoprenoid biosyn- 


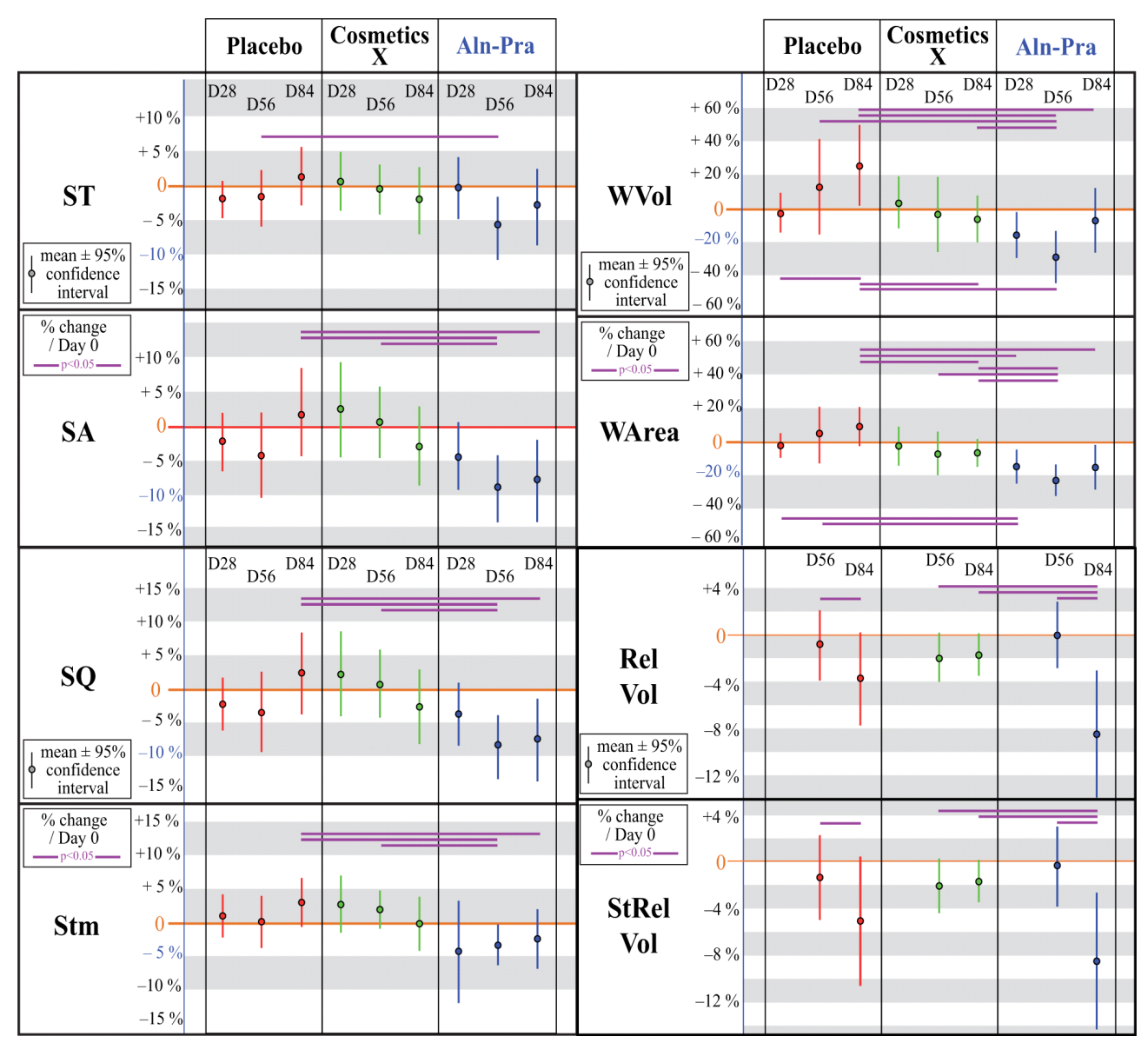

Figure 3. Changes in rugosity and morphological parameters induced by topical cosmetic treatments. Rugosity and morphological parameters (crow's feet): [D28-D0]/D0, [D56-D0]/D0, [D84-D0]/D0 expressed as percentages. ST (maximum amplitude of the relief). SA (average roughness). SQ (average roughness with regard to the average quadratic variation). Stm (mean difference between peaks and valley). Vol (volume of the main wrinkle). Ar (area of the main wrinkle). Morphological parameters (cheek): [D56-D0]/D0, [D84-D0]/D0 expressed as percentages. relVol (relative volume of the hollow cheek). StrelVol (standardized relative volume of the hollow cheek). Parameter mean $\pm 95 \%$ confidence interval. Horizontal pink lines: statistically significant difference $(p<0.05)$.

thesis pathways in mesenchymal cells [43]. Pravastatin has been shown to induce collagen synthesis and to modulate the expression of MMPs and of their TIMP inhibitors in blood vessel walls $[44,45]$.

Because statins and aminobisphosphonates exert a synergistic effect on bone and cartilage cells, and on bone and cartilage extracellular matrices [46], our alendronatepravastatin topical combination probably elicited similar effects on human skin, thus explaining the anti-wrinkle activity observed in our study.

Progerin has already been detected in both keratinocytes and dermal fibroblasts in human skin biopsies sampled from several anatomical regions of aged patients [11]. Furthermore, the combination of another aminobisphosphonate (zoledronate) and pravastatin restores several morphological parameters (nuclear shape and size) and numerous biological parameters (DNA repair, over- expression of p53 target genes) in cultured fibroblasts from progeria patient skin biopsies, as well as skin defects in a progeria mouse model [12]. The same zoledronate-pravastatin combination corrects the shape of skin keratinocyte nuclei in model mice expressing progerin under the control of the keratin 14 promoter [47]. Through the inhibition of two enzymes of the isoprenoid biosynthesis pathway, HMG-CoA reductase and farnesylpyrophosphate synthase, respectively, statin and aminobisphosphonate decreased the synthesis of farnesylpyrophosphate, the precursor of the farnesyl anchor on proteins [7]. Mass spectrometry experiments have demonstrated that progerin is neither farnesylated nor geranylgeranylated in skin fibroblasts from progeria patients or from a mouse model of progeria, treated with zoledronate and pravastatin [12]. Thus, the zoledronate-pravastatin combination reduces the cellular toxicity and pro-aging 
D0
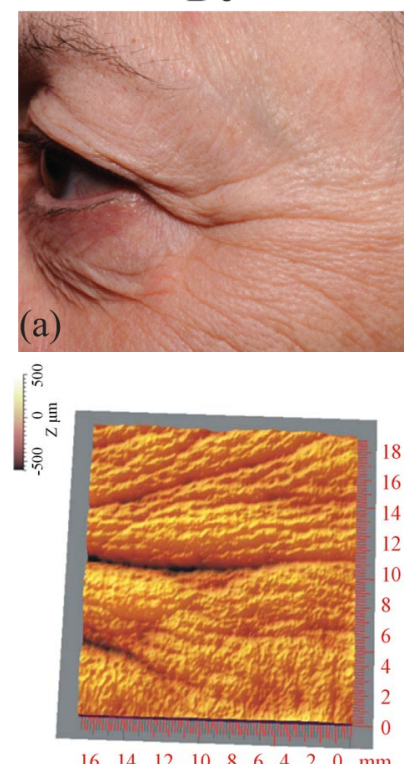

(c)
D84
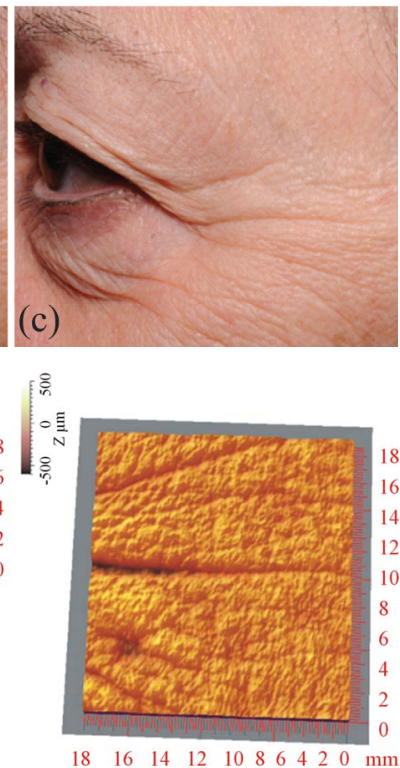

(d)
Figure 4. Improvement of wrinkle micro-relief by the alendronate-pravastatin topical combination. Original pictures before (D0, a) and after 84 days of treatment (D84, c). Digitized and flattened pictures before (b) and after treatment (d). Changes in crow's feet rugosity and morphological parameters (expressed as percentages) from one subject [ma ximal change; minimal change recorded in the whole subject sample]. ST $-22.7 \%[-28.2 \% ; 17.1 \%]$. SA $-41.1 \%$ $[-41.1 \% ; 15.3 \%]$. SQ $-40.6 \% \quad[-40.6 \% ; 17.4 \%]$. Stm $-28.7 \%$ [-28.7\%; 19.5\%]. Vol $-60.4 \%$ [-74.4\%; $109.0 \%]$. $\mathrm{Ar}-\mathbf{4 1 . 1 \%}[-\mathbf{5 0 . 8 \%} ; \mathbf{2 5 . 0 \%}]$.

activity of progerin [12].

\section{Conclusion}

In conclusion, the present study showed that a cosmetic preparation containing a combination of the aminobisphosphonate, alendronate, and pravastatin exhibited antiaging properties on human skin.

\section{Acknowledgements}

We thank the volunteers who participated and Spincontrol who conducted the study, which was funded by PrenylB Company. Bénédicte Cantecor was the recipient of a grant from French National Association for Research and Technology (ANRT). We thank Sophie Perrin for carefully reading the manuscript and APL Scientific for English editing.

\section{REFERENCES}

[1] M. A. Farage, K. W. Miller and H. I. Maibach, "Degenerative Changes in Aging Skin,” In: M. A. Farage, K. W.
Miller and H. I. Maibach, Eds., Textbook of Aging Skin, Springer, Berlin, 2010, pp. 25-35. doi:10.1007/978-3-540-89656-2_4

[2] D. L. Bissett and M. B. Johnson, "Cosmetic Anti-Aging Ingredients,” In: M. A. Farage, K. W. Miller and H. I. Maibach, Eds., Textbook of Aging Skin, Springer, Berlin, 2010, pp. 1069-1078. doi:10.1007/978-3-540-89656-2 99

[3] R. C. Hennekam, "Hutchinson-Gilford Progeria Syndrome: Review of the Phenotype," American Journal of Medical Genetics Part A, Vol. 140, No. 23, 2006, pp. 2603-2624. doi:10.1002/ajmg.a.31346

[4] M. A. Merideth, L. B. Gordon, S. Clauss, V. Sachdev, A. C. Smith, M. B. Perry, et al., "Phenotype and Course of Hutchinson-Gilford Progeria Syndrome," The New England Journal of Medicine, Vol. 358, No. 6, 2008, pp. 592604. doi:10.1056/NEJMoa0706898

[5] A. De Sandre-Giovannoli, R. Bernard, P. Cau, C. Navarro, J. Amiel, I. Boccaccio, et al., "Lamin a Truncation in $\mathrm{Hu}-$ tchinson-Gilford Progeria,” Science, Vol. 300, No. 5628, 2003, p. 2055. doi:10.1126/science. 1084125

[6] M. Eriksson, W. T. Brown, L. B. Gordon, M. W. Glynn, J. Singer, L. Scott, et al., "Recurrent de Novo Point Mutations in Lamin A Cause Hutchinson-Gilford Progeria Syndrome," Nature, Vol. 423, No. 6937, 2003, pp. 293-298. doi:10.1038/nature01629

[7] C. L. Navarro, P. Cau and N. Levy, "Molecular Bases of Progeroid Syndromes," Human Molecular Genetics, Vol. 15, Suppl. 2, 2006, pp. 151-161. doi:10.1093/hmg/ddl214

[8] P. Scaffidi and T. Misteli, "Lamin A-Dependent Nuclear Defects in Human Aging,” Science, Vol. 312, No. 5776, 2006, pp. 1059-1063. doi:10.1126/science.1127168

[9] J. Espada, I. Varela, I. Flores, A. P. Ugalde, J. Cadinanos, A. M. Pendas, et al., "Nuclear Envelope Defects Cause Stem Cell Dysfunction in Premature-Aging Mice," The Journal of Cell Biology, Vol. 181, No. 1, 2008, pp. 27-35. doi:10.1083/jcb.200801096

[10] P. Scaffidi and T. Misteli, "Lamin A-Dependent Misregulation of Adult Stem Cells Associated with Accelerated Ageing,” Nature Cell Biology, Vol. 10, No. 4, 2008, pp. 452-459. doi:10.1038/ncb1708

[11] D. McClintock, D. Ratner, M. Lokuge, D. M. Owens, L. B. Gordon, F. S. Collins, et al., "The Mutant form of Lamin A that Causes Hutchinson-Gilford Progeria Is a Biomarker of Cellular Aging in Human Skin,” PLoS One, Vol. 2, No. 12, 2007, Article ID: e1269. doi:10.1371/journal.pone.0001269

[12] I. Varela, S. Pereira, A. P. Ugalde, C. L. Navarro, M. F. Suarez, P. Cau, et al., "Combined Treatment with Statins and Aminobisphosphonates Extends Longevity in a Mouse Model of Human Premature Aging," Nature Medicine, Vol. 14, No. 7, 2008, pp. 767-772. doi:10.1038/nm1786

[13] B. Cantecor, M. P. Savelli, G. Marti-Mestres, V. Bonniol, M. A. Mostefa Side Larbi and P. Piccerelle, "Recent Advances in Topical Applications for a New Anti-Aging Drug,” In: R. Chilcott and K. R. Brain, Eds., Advances in Dermatological Sciences, Royal Society of Chemistry, London, 2013. 
[14] R. Bazin and E. Doublet, “Atlas du Vieillissementcutané: Volume 1, Population Européenne,” Med’Com, 2007.

[15] D. Karadzovska, J. D. Brooks, N. A. Monteiro-Riviere and J. E. Riviere, "Predicting Skin Permeability from Complex Vehicles,” Advanced Drug Delivery Reviews, Vol. 65, No. 2, 2012, pp. 265-277. doi:10.1016/j.addr.2012.01.019

[16] M. Rohr and K. Schrader, "Fast Optical in Vivo Topometry of Human Skin (FOITS): Comparative Investigation with Laser Profilometry,” SOFW Journal, Vol. 124, No. 2, 1998, pp. 52-59.

[17] M. Rohr, M. Brandt and K. Schrader, "Skin Surface Claim Supported by FOITS,” SOFW Journal, Vol. 126, No. 8, 2000, pp. 2-11.

[18] C. J. Huberty and S. Olejnik, "Applied MANOVA and Discriminant Analysis," Wiley-Interscience, Hoboken, 2006. doi:10.1002/047178947X

[19] A. Choi, H. Gang, I. Chun and H. Gwak, "The Effects of Fatty Acids in Propylene Glycol on the Percutaneous Absorption of Alendronate across the Excised Hairless Mouse Skin," International Journal of Pharmaceutics, Vol. 357, No. 1-2, 2008, pp. 126-131. doi:10.1016/j.ijpharm.2008.01.050

[20] C. A. Scott, D. Tattersall, E. A. O’Toole and D. P. Kelsell, "Connexins in Epidermal Homeostasis and Skin Disease,” Biochimica et Biophysica Acta, Vol. 1818, No. 8, 2012, pp. 1952-1961. doi:10.1016/j.bbamem.2011.09.004

[21] K. E. Moyer and H. P. Ehrlich, "Modulation of Human Fibroblast Gap Junction Intercellular Communication by Hyaluronan,” Journal of Cellular Physiology, Vol. 196, No. 1, 2003, pp. 165-170. doi:10.1002/jcp.10288

[22] H. M. Langevin, C. J. Cornbrooks and D. J. Taatjes, "Fibroblasts form a Body-Wide Cellular Network," Histochemistry and Cell Biology, Vol. 122, No. 1, 2004, pp. 7-15. doi:10.1007/s00418-004-0667-z

[23] T. Bellido and L. I. Plotkin, "Novel Actions of Bisphosphonates in Bone: Preservation of Osteoblast and Osteocyte Viability,” Bone, Vol. 49, No. 1, 2011, pp. 50-55. doi:10.1016/j.bone.2010.08.008

[24] M. Sato, W. Grasser, N. Endo, R. Akins, H. Simmons, D. D. Thompson, et al., "Bisphosphonate Action. Alendronate Localization in Rat Bone and Effects on Osteoclast Ultrastructure," The Journal of Clinical Investigation, Vol. 88, No. 6, 1991, pp. 2095-2105. doi:10.1172/JCI115539

[25] M. J. Rogers, S. Gordon, H. L. Benford, F. P. Coxon, S. P. Luckman, J. Monkkonen, et al., "Cellular and Molecular Mechanisms of Action of Bisphosphonates," Cancer, Vol. 88, Suppl. 12, 2000, pp. 2961-2978. doi:10.1002/1097-0142(20000615)88:12+<2961::AID-C NCR12>3.0.CO;2-L

[26] E. van Beek, E. Pieterman, L. Cohen, C. Löwik and S. Papapoulos, "Farnesyl Pyrophosphate Synthase Is the Molecular Target of Nitrogen-Containing Bisphosphonates," Biochemical and Biophysical Research Communications, Vol. 264, No. 1, 1999, pp. 108-111. doi:10.1006/bbrc.1999.1499
[27] J. D. Bergstrom, R. G. Bostedor, P. J. Masarachia, A. A. Reszka and G. Rodan, "Alendronate Is a Specific, Nanomolar Inhibitor of FarnesylDiphosphate Synthase,” Archives of Biochemistry and Biophysics, Vol. 373, No. 1, 2000, pp. 231-241. doi:10.1006/abbi.1999.1502

[28] M. Xue and C. J. Jackson, “Autocrine Actions of Matrix Metalloproteinase (MMP)-2 Counter the Effects of MMP9 to Promote Survival and Prevent Terminal Differentiation of Cultured Human Keratinocytes," Journal of Investigative Dermatology, Vol. 128, No. 11, 2008, pp. 2676-2685. doi:10.1038/jid.2008.136

[29] A. A. Tandara and T. A. Mustoe, "MMP- and TIMP-Secretion by Human Cutaneous Keratinocytes and Fibroblasts-Impact of Coculture and Hydration,” Journal of Plastic, Reconstructive \& Aesthetic Surgery, Vol. 64, No. 1, 2011, pp. 108-116. doi:10.1016/j.bjps.2010.03.051

[30] S. Malgouries, S. Thibaut and B. A. Bernard, "Proteoglycan Expression Patterns in Human Hair Follicle,” British Journal of Dermatology, Vol. 158, No. 2, 2008, pp. 234-242. doi:10.1111/j.1365-2133.2007.08339.x

[31] R. V. Iozzo, "Matrix Proteoglycans: From Molecular Design to Cellular Function,” Annual Review of Biochemistry, Vol. 67, 1998, pp. 609-652.

[32] H. Jarvelainen, A. Sainio, M. Koulu, T. N. Wight and R. Penttinen, "Extracellular Matrix Molecules: Potential Targets in Pharmacotherapy,” Pharmacological Reviews, Vol. 61, No. 2, 2009, pp. 198-223. doi:10.1124/pr.109.001289

[33] Y. Shitara and Y. Sugiyama, "Pharmacokinetic and Pharmacodynamic Alterations of 3-Hydroxy-3-Methylg-lutaryl Coenzyme A (HMG-CoA) Reductase Inhibitors: Drug-Drug Interactions and Interindividual Differences in Transporter and Metabolic Enzyme Functions,” Pharmacology \& Therapeutics, Vol. 112, No. 1, 2006, pp. 71-105. doi:10.1016/j.pharmthera.2006.03.003

[34] K. M. Giacomini, S. M. Huang, D. J. Tweedie, L. Z. Benet, K. L. Brouwer, X. Chu, et al., "Membrane Transporters in Drug Development," Nature Reviews Drug Discovery, Vol. 9, No. 3, 2010, pp. 215-236. doi:10.1038/nrd3028

[35] M. Roth, A. Obaidat and B. Hagenbuch, “OATPs, OATs and OCTs: The Organic Anion and Cation Transporters of the SLCO and SLC22A Gene Superfamilies,” British Journal of Pharmacology, Vol. 165, No. 5, 2012, pp. 1260-1287. doi:10.1111/j.1476-5381.2011.01724.x

[36] B. Hagenbuch and P. J. Meier, "The Superfamily of Organic Anion Transporting Polypeptides,” Biochimica et Biophysica Acta, Vol. 1609, No. 1, 2003, pp. 1-18. doi:10.1016/S0005-2736(02)00633-8

[37] R. Schiffer, M. Neis, D. Holler, F. Rodriguez, A. Geier, C. Gartung, et al., “Active Influx Transport Is Mediated by Members of the Organic Anion Transporting Polypeptide Family in Human Epidermal Keratinocytes," Journal of Investigative Dermatology, Vol. 120, No. 2, 2003, pp. 285-291. doi:10.1046/j.1523-1747.2003.12031.x

[38] G. D. Kruh and M. G. Belinsky, "The MRP Family of Drug Efflux Pumps,” Oncogene, Vol. 22, No. 47, 2003, pp. 7537-7552. doi:10.1038/sj.onc. 1206953 
[39] Z.-S. Chen and A. K. Tiwari, "Multidrug Resistance Proteins (MRPs/ABCCs) in Cancer Chemotherapy and Genetic Diseases,” FEBS Journal, Vol. 278, No. 18, 2011, pp. 3226-3245. doi:10.1111/j.1742-4658.2011.08235.x

[40] D. Hendig, T. Langmann, S. Kocken, R. Zarbock, C. Szliska, G. Schmitz, et al., "Gene Expression Profiling of ABC Transporters in Dermal Fibroblasts of Pseudoxanthomaelasticum Patients Identifies New Candidates Involved in PXE Pathogenesis,” Laboratory Investigation, Vol. 88, No. 12, 2008, pp. 1303-1315. doi:10.1038/labinvest.2008.96

[41] L. Xing, Y. Hu and Y. Lai, "Advancement of StructureActivity Relationship of Multidrug Resistance-Associated Protein 2 Interactions," AAPS Journals, Vol. 11, No. 3, 2009, pp. 406-413. doi:10.1208/s12248-009-9117-0

[42] F. Jowkar and M. R. Namazi, "Statins in Dermatology," International Journal of Dermatology, Vol. 49, No. 11, 2010, pp. 1235-1243. doi:10.1111/j.1365-4632.2010.04579.X

[43] F. M. Sverdrup, M. P. Yates, L. E. Vickery, J. A. Klover, L. R. Song, C. P. Anglin, et al., "Protein Geranylgeranylation Controls Collagenase Expression in Osteoarthritic Cartilage,” Osteoarthritis Cartilage, Vol. 18, No. 7, 2010, pp. 948-955. doi:10.1016/j.joca.2010.03.015
[44] M. Crisby, G. Nordin-Fredriksson, P. K. Shah, J. Yano, J. Zhu and J. Nilsson, "Pravastatin Treatment Increases Collagen Content and Decreases Lipid Content, Inflammation, Metalloproteinases, and Cell Death in Human Carotid Plaques: Implications for Plaque Stabilization," Circulation, Vol. 103, No. 7, 2001, pp. 926-933. doi:10.1161/01.CIR.103.7.926

[45] Y. Fukumoto, P. Libby, E. Rabkin, C. C. Hill, M. Enomoto, Y. Hirouchi, et al., "Statins Alter Smooth Muscle Cell Accumulation and Collagen Content in Established Atheroma of Watanabe Heritable Hyperlipidemic Rabbits," Circulation, Vol. 103, No. 7, 2001, pp. 993-999. doi:10.1161/01.CIR.103.7.993

[46] H. Mo, H. Yeganehjoo, A. Shah, W. K. Mo, I. N. Soelaiman and C. L. Shen, "Mevalonate-Suppressive Dietary Isoprenoids for Bone Health,” The Journal of Nutritional Biochemistry, Vol. 23, No. 12, 2012, pp. 1543-1551. doi:10.1016/j.jnutbio.2012.07.007

[47] Y. Wang, C. Ostlund and H. J. Worman, "Blocking Protein Farnesylation Improves Nuclear Shape Abnormalities in Keratinocytes of Mice Expressing the Prelamin A Variant in Hutchinson-Gilford Progeria Syndrome,” Nucleus, Vol. 1, No. 5, 2010, pp. 432-439. 